\title{
Light trapping in amorphous silicon solar cells with periodic grating structures
}

\author{
Haihua Li ${ }^{\text {a }}$, Qingkang Wang ${ }^{\mathrm{a}, *}$, Jian Chen ${ }^{\mathrm{a}}$, Janez $\mathrm{Krc}^{\mathrm{b}}$, Wim J. Soppe ${ }^{\mathrm{c}}$

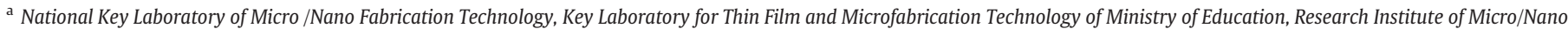 \\ Science and Technology, Shanghai Jiao Tong University, Shanghai 200240, China \\ ${ }^{\mathrm{b}}$ University of Ljubljana, Faculty of Electrical Engineering, Trzaska25, 1000 Ljubljana, Slovenia \\ ' Energy research Center of the Netherlands, P. O. Box 1, 1755 ZG Pettern, The Netherlands
}

\section{A R T I C L E I N F O}

Article history:

Received 31 December 2010

Received in revised form 23 September 2011

Accepted 7 November 2011

Available online 24 November 2011

\section{Keywords:}

Thin-film silicon solar cell

Anti-reflection structure

Metallic reflection grating

Rigorous Coupled Wave Analysis

\begin{abstract}
A B S T R A C T
We report on the design of amorphous silicon solar cells with the periodic grating structures. It is a combination of an anti-reflection structure and the metallic reflection grating. Optical coupling and light trapping in thin-film solar cells are studied numerically using the Rigorous Coupled Wave Analysis enhanced by the Modal Transmission Line theory. The impact of the structure parameters of the gratings is investigated. The results revealed that within the incident angles of $-40^{\circ}$ to $+40^{\circ}$ the reflectivity of the cell with a period of $0.5 \mu \mathrm{m}$, a filling factor of 0.1 and a groove depth of $0.4 \mu \mathrm{m}$ is $4 \%-22.7 \%$ in the wavelength range of $0.3-0.6 \mu \mathrm{m}$ and $1 \%-20.8 \%$ in the wavelength range of $0.6-0.84 \mu \mathrm{m}$, the absorption enhancement of the a-Si layer is $0.4 \%-10.8 \%$ and $20 \%-385 \%$, respectively.
\end{abstract}

(c) 2011 Elsevier B.V. All rights reserved.

\section{Introduction}

Thin-film silicon (TF-Si) solar cell based on amorphous silicon (a$\mathrm{Si}: \mathrm{H}$ ) has gained considerable attention as promising candidate for future generation of photovoltaics [1,2]. In the past, in most cases glass or metal sheets were used as substrate materials for amorphous silicon solar cells. For further reductions of manufacturing cost, plastic or steel foil could turn out to be more suitable substrate. Regarding costs, the use of foil allows one to combine both roll-to-roll continuous fabrication, and an easy possibility of direct monolithic series connection [3-5].

In TF-Si solar cells, efficient light management is essential in order to obtain sufficiently high photocurrent from the thin absorber layers in the cells (thickness from $0.2 \mu \mathrm{m}$ to a few $\mu \mathrm{m}$ ). The key solution is to enhance the optical path length by introducing efficient light trapping in the cell structures. Light trapping is the standard technique for harvesting the incoming sunlight to improve the TF-Si cell efficiencies. Introducing textured interfaces can lead to reduced reflection losses and enhanced scattering and diffraction of light in the device.

In Fig. 1(a) the schematic cross-section of a flat TF-Si solar cell is shown. The schematic cross-section of our designed solar cell structure is shown in Fig. 1(b). We first introduce the dielectric gratings on the foil substrate, then silver back contact $(100 \mathrm{~nm}), \mathrm{ZnO}(40 \mathrm{~nm})$, n-a-Si:H (20 nm), i-a-Si:H absorber (200 nm), p-a-Si:H (15 nm), and ITO front transparent conductive oxide contact $(70 \mathrm{~nm})$. The gratings can be imprinted directly by hot embossing in a plastic

\footnotetext{
* Corresponding author.

E-mail address: wangqingkang@sjtu.edu.cn (Q. Wang).
}

substrate, or in a barrier layer in case of the steel foil $[6,7,8]$. The advantage of this structure will be revealed in terms of an antireflection effect at the front interfaces and efficient light scattering at the metallic reflection grating and other textured interfaces. Each dielectric layer can be seen as an anti-reflection grating, the silver film can be seen as metallic diffraction grating in the role of back reflector and diffuser at the same time.

For reducing the metallic loss we insert a $\mathrm{ZnO}$ layer between the a-Si layer and the metallic diffraction gratings $[9,10]$. Transparent $\mathrm{ZnO}$ can be used for TF-Si solar cells because of its superior transparency and stronger resistance to a hydrogen plasma. Because $\mathrm{ZnO}$ has a lower extinction coefficient (less than 0.5 ) in the longer wavelength region, due to the insertion of a thin $\mathrm{ZnO}$ layer in the a-Si/Ag interface, the absorption loss is greatly minimized. ZnO layer not only can reduce the metallic loss, but also enhance the back reflection. When the angle of incidence of the ray at the a-Si/ZnO interface is greater than the critical angle between a-Si and the $\mathrm{ZnO}$ layers, light will be totally internally reflected, and the effective path length will be increased due to multiple reflections [11].

In this work, simulations are utilized to investigate he reflectivity of the entire TF-Si solar cell structure to find out the optimal structure parameters of the grating on substrate, which are shown in Fig. 1(b): the period (a), the filling factor (f), and the height of the grating ( $h$ ). The aim is to minimize the total reflectance of the solar cell, for various incident angles of illumination by optimizing the grating parameters. Minimal reflectance consequently means improved light trapping in the solar cell structure and thus stronger absorption of light inside the cell. However, the enhanced absorption in the cell can't be fully assigned to the absorption in the a-Si: layer, leading to increased photocurrent, but also increased optical losses in supporting layers (like front and 
(a)

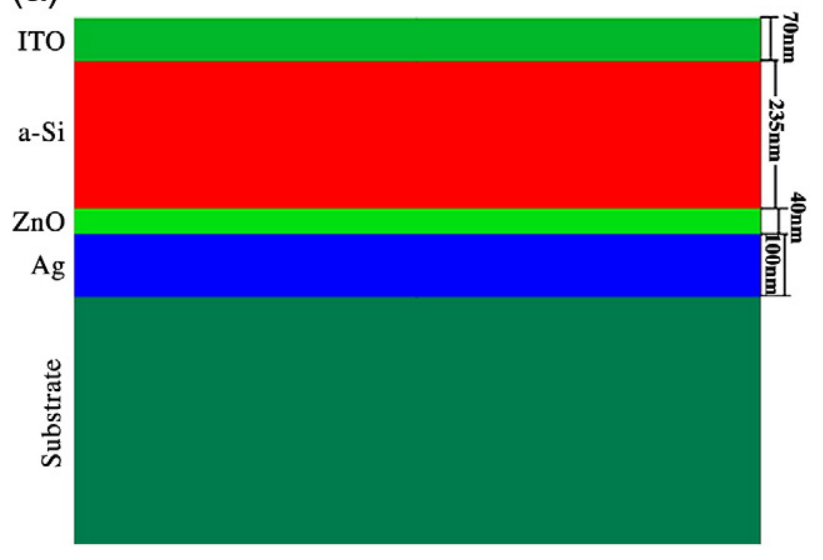

(b)

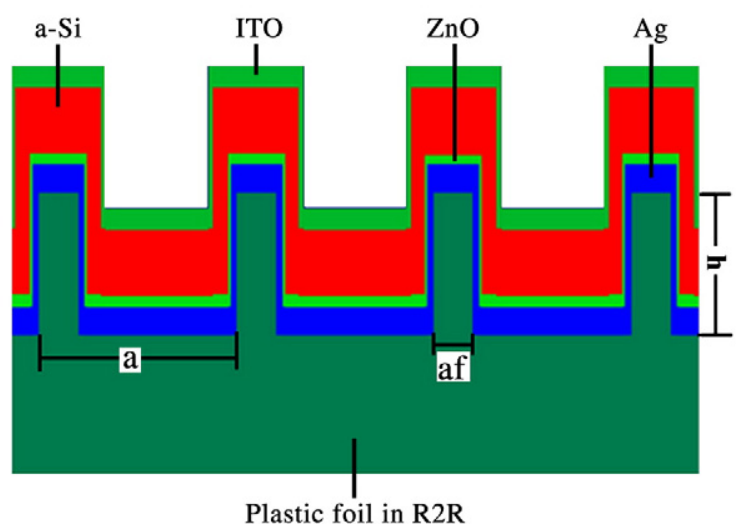

Fig. 1. (a) a flat TF-Si solar cell (b) the designed TF-Si solar cell structure.

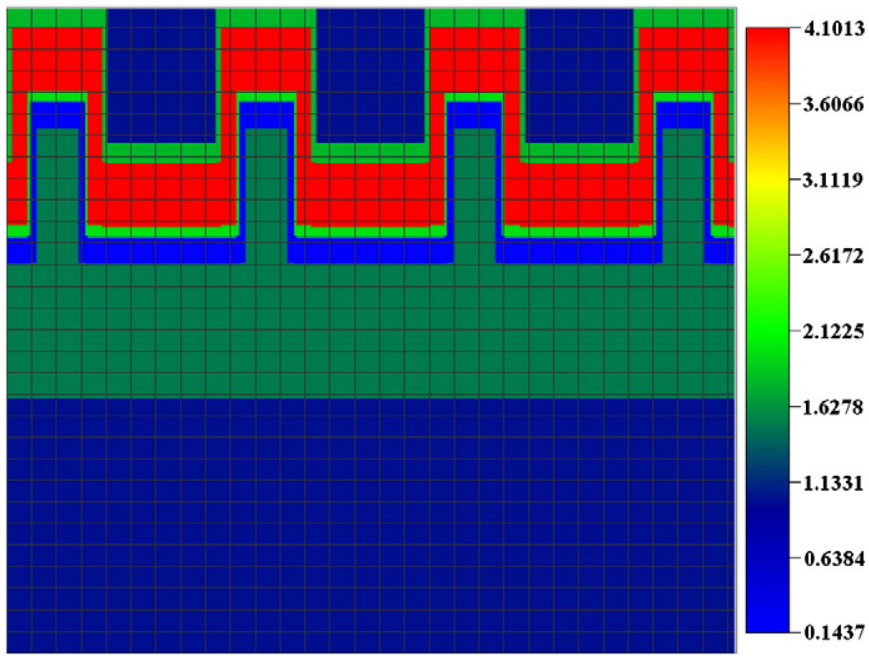

Fig. 2. The refractive index distribution of the TF-Si solar cell at $\lambda=0.8 \mu \mathrm{m}$.

back contact). So the absorption enhancement of the a-Si solar cell also considered.

\section{Principle and numerical modeling}

In our approach, the core algorithm, which is based on the Rigorous Coupled Wave Analysis (RCWA) [12] enhanced by the Modal Transmission Line (MTL) theory, is used to find the solution of Maxwell's equations for the light propagation in the structure. The calculation of reflectance and absorption includes three steps: (1) obtaining the expressions of electromagnetic field in incident region and transmitted region, (2) Fourier expansion of electromagnetic field and dielectric constant in the TF-Si solar cell, (3) using boundary condition for electromagnetic field at different interface, obtaining the amplitude and the diffraction efficiency of different levels by some mathematical methods. The reflectance is for all the powers from all of the reflected diffraction orders added together. The absorption is for all the powers from all of the absorbed diffraction orders added together.

For $2 \mathrm{D}$ or $3 \mathrm{D}$ structures with arbitrary incident directions, the vectorized forms of the equations are taken into account. By factoring out an assumed time harmonic factor $\exp (-i \omega t)$, Maxwell's equations can be expressed as the following form

$\frac{\partial}{\partial y} E_{z}-\frac{\partial}{\partial z} E_{y}=i \omega \mu H_{x}$

$\frac{\partial}{\partial z} E_{x}-\frac{\partial}{\partial x} E_{z}=i \omega \mu H_{y}$

$\frac{\partial}{\partial x} E_{y}-\frac{\partial}{\partial y} E_{x}=i \omega \mu H_{z}$

$\frac{\partial}{\partial y} H_{z}-\frac{\partial}{\partial z} H_{y}=-i \omega \varepsilon_{0} \varepsilon_{r, x} E_{x}$

$\frac{\partial}{\partial z} H_{x}-\frac{\partial}{\partial x} H_{z}=-i \omega \varepsilon_{0} \varepsilon_{r, y} E_{y}$

$\frac{\partial}{\partial x} H_{y}-\frac{\partial}{\partial y} H_{x}=-i \omega \varepsilon_{0} \varepsilon_{r, z} E_{z}$

where $t$ is the time, $\omega$ is the angular frequency, $\varepsilon_{0}$ is the vacuum permittivity, $\mu$ is magnetic permeability of the material, $E$ is electric field intensity and $H$ is magnetic field intensity. The medium is characterized by a diagonal index tensor with respect to the principal axes with diagonal elements: $\varepsilon_{r, x}, \varepsilon_{r, y}, \varepsilon_{r, z}$.

Table 1

The complex refractive index of the materials with different wavelengths.

\begin{tabular}{|c|c|c|c|c|c|c|c|c|c|c|}
\hline & & $\lambda=0.45 \mu \mathrm{m}$ & $\lambda=0.5 \mu \mathrm{m}$ & $\lambda=0.55 \mu \mathrm{m}$ & $\lambda=0.6 \mu \mathrm{m}$ & $\lambda=0.65 \mu \mathrm{m}$ & $\lambda=0.7 \mu \mathrm{m}$ & $\lambda=0.75 \mu \mathrm{m}$ & $\lambda=0.8 \mu \mathrm{m}$ & $\lambda=0.84 \mu \mathrm{m}$ \\
\hline \multirow[t]{2}{*}{ ITO } & $n$ & 2.03596 & 1.97235 & 1.92156 & 1.88081 & 1.84783 & 1.82088 & 1.79863 & 1.78008 & 1.76739 \\
\hline & $\kappa$ & 0.040563 & 0.04309 & 0.048057 & 0.054006 & 0.060216 & 0.06633 & 0.07218 & 0.077688 & 0.081832 \\
\hline \multirow[t]{2}{*}{$\mathrm{a}-\mathrm{Si}$} & $n$ & 5.1197 & 4.9796 & 4.7747 & 4.6237 & 4.4643 & 4.3199 & 4.2088 & 4.1013 & 4.0454 \\
\hline & $\kappa$ & 1.3954 & 0.89 & 0.5213 & 0.3267 & 0.1872 & 0.0989 & 0.0522 & 0.0297 & 0.0219 \\
\hline $\mathrm{ZnO}$ & $n$ & 2.1054 & 2.0516 & 2.01976 & 1.99891 & 1.98433 & 1.97362 & 1.96547 & 1.95908 & 1.9549 \\
\hline \multirow[t]{2}{*}{$\mathrm{Ag}$} & $n$ & 0.15129 & 0.13 & 0.125 & 0.12425 & 0.13928 & 0.14221 & 0.14573 & 0.14397 & 0.14909 \\
\hline & $\kappa$ & 2.47024 & 2.91763 & 3.33898 & 3.73159 & 4.12853 & 4.52296 & 4.89888 & 5.28905 & 5.6022 \\
\hline
\end{tabular}




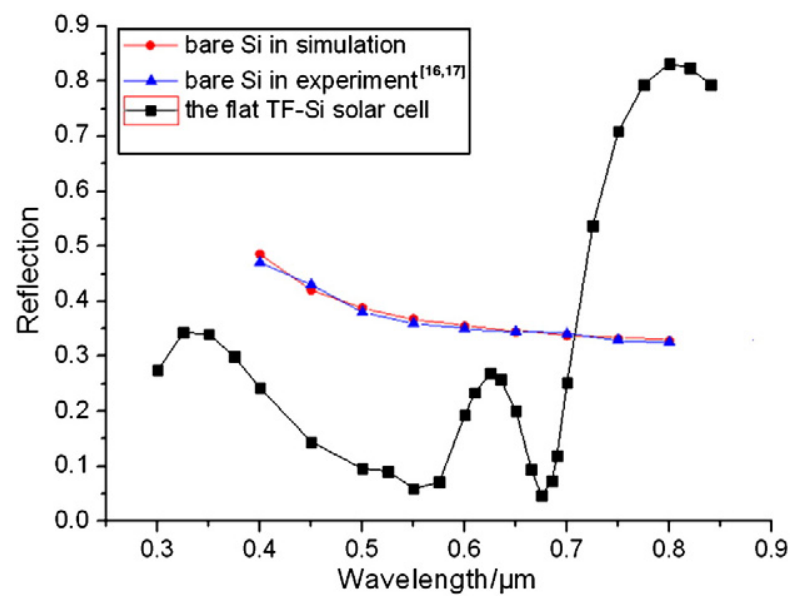

Fig. 3. The measured and simulated reflectivity of a bare silicon wafer. The reflectivity of the flat TF-Si solar cell in Fig. 1(a) as a function of incident wavelength for normal incidence.

By substituting Eqs. (1c), (1f) into Eqs. (1a), (1b), (1d), (1e), we derive the following transverse format of Maxwell's equations:

$\frac{\partial}{\partial z} E_{x}=\frac{-i}{\omega \varepsilon_{0}} \frac{\partial}{\partial x} \frac{1}{\varepsilon_{r, z}} \frac{\partial}{\partial y} H_{x}+\left(\frac{i}{\omega \varepsilon_{0}} \frac{\partial}{\partial x} \frac{1}{\varepsilon_{r, z}} \frac{\partial}{\partial x}+i \omega \mu\right) H_{y}$
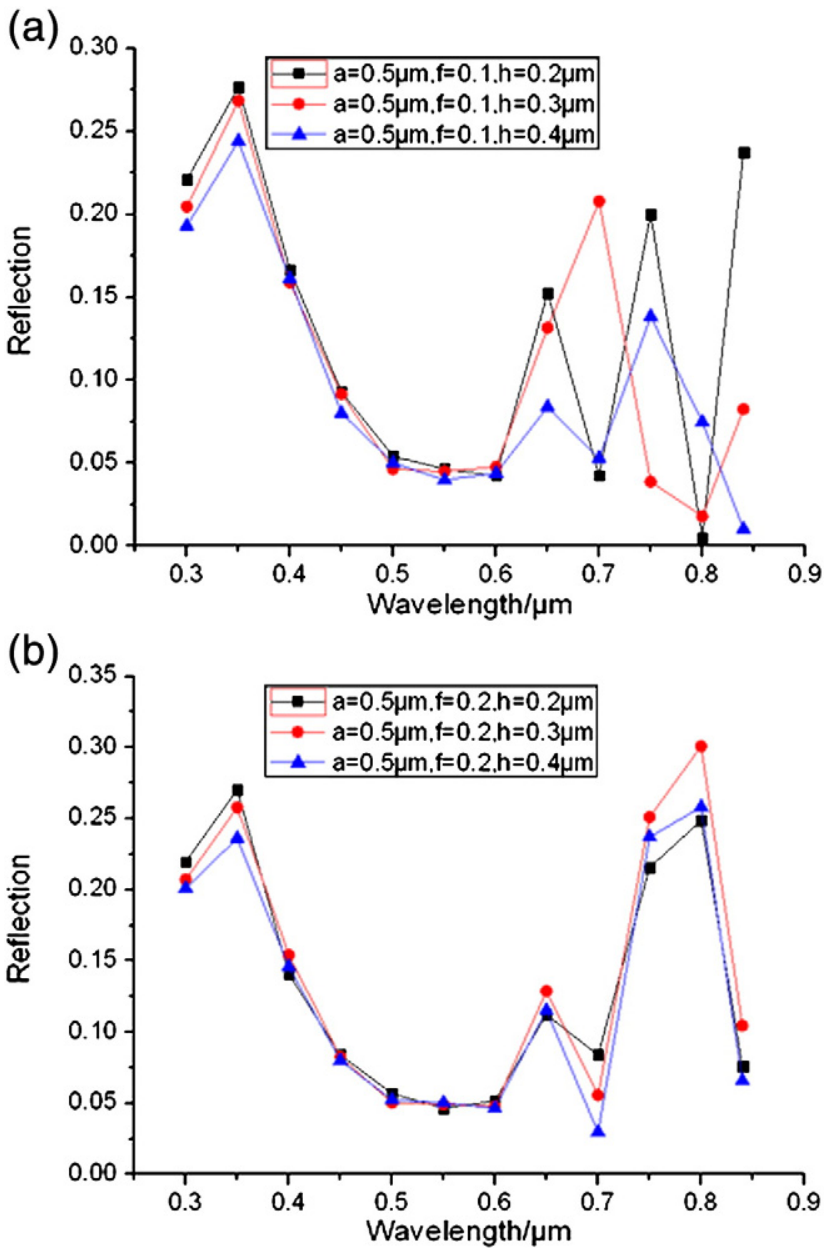

Fig. 4. The reflectivity of the designed TF-Si solar cell as a function of the incident wavelength with the same $a=0.5 \mu \mathrm{m}$, but with different $f$ and $h$ for normal incidence.
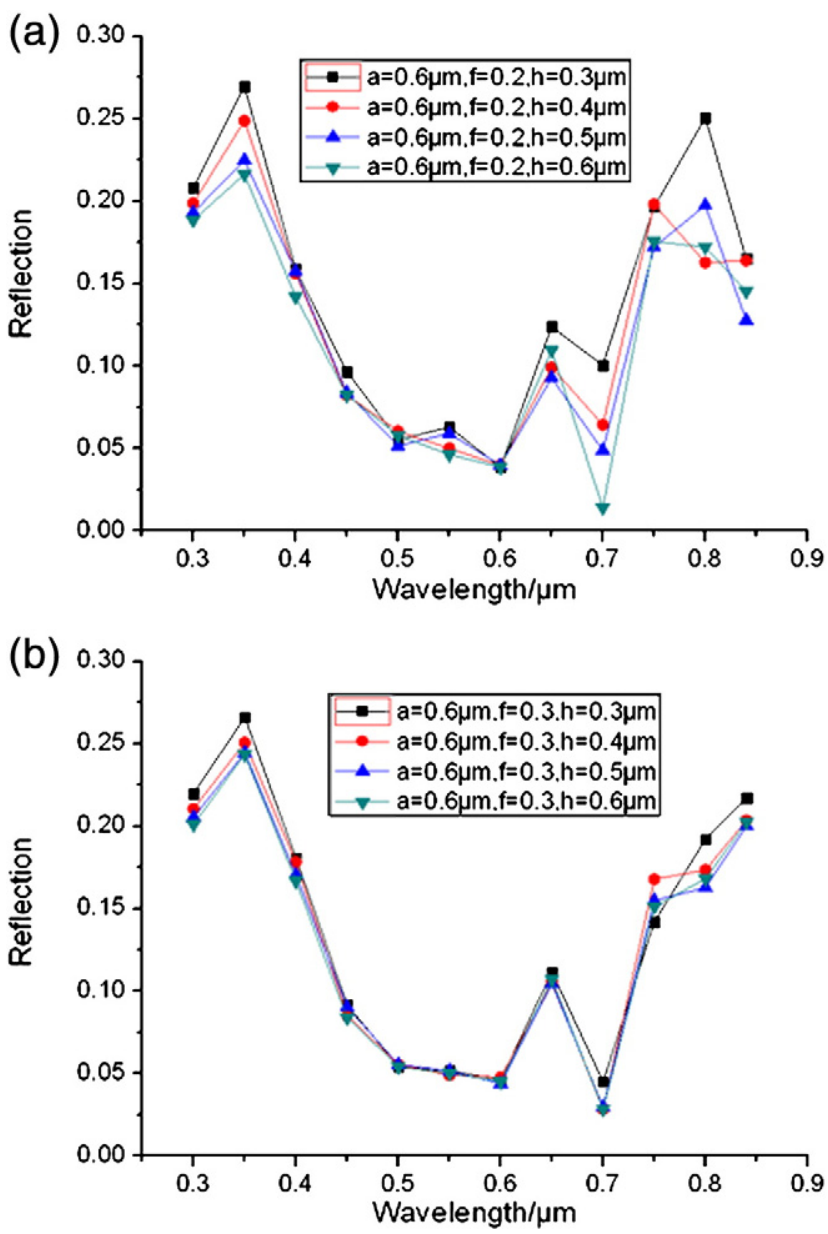

Fig. 5. The reflectivity of the designed TF-Si solar cell as a function of the incident wavelength with the same $a=0.6 \mu \mathrm{m}$, but different $f$ and $h$ for normal incidence.

$$
\begin{aligned}
\frac{\partial}{\partial z} E_{y} & =\left(\frac{-i}{\omega \varepsilon_{0}} \frac{\partial}{\partial y} \frac{1}{\varepsilon_{r, z}} \frac{\partial}{\partial y}-i \omega \mu\right) H_{x}+\frac{i}{\omega \varepsilon_{0}} \frac{\partial}{\partial y} \frac{1}{\varepsilon_{r, z}} \frac{\partial}{\partial x} H_{y} \\
\frac{\partial}{\partial z} H_{x} & =\frac{i}{\omega \mu} \frac{\partial}{\partial x} \frac{\partial}{\partial y} E_{x}+\left(\frac{-i}{\omega \mu} \frac{\partial}{\partial x} \frac{\partial}{\partial x}-i \omega \varepsilon_{0} \varepsilon_{r, y}\right) E_{y} \\
\frac{\partial}{\partial z} H_{y} & =\left(\frac{i}{\omega \mu} \frac{\partial}{\partial y} \frac{\partial}{\partial y}+i \omega \varepsilon_{0} \varepsilon_{r, x}\right) E_{x}+\frac{-i}{\omega \mu} \frac{\partial}{\partial y} \frac{\partial}{\partial x} E_{y}
\end{aligned}
$$

For scattering problems, we want to calculate the reflected and transmitted light waves from the incident field. A direct solution in the spatial domain of Eq. (2) with proper boundary conditions is computationally expensive. Moreover, in order to calculate accurate diffraction efficiencies for all diffraction orders, fine simulation grids are essential. Since the RCWA and MTL methods are based on the Fourier domain, they are efficient solutions for the amplitude of each diffraction order. In order to solve these equations, we need to break the grating structure into simple building blocks with a vertically homogenous region, i.e., a region with values of $\varepsilon_{r, x}, \varepsilon_{r, y}$ and $\varepsilon_{r, z}$, which are independent of $z$. A complicated multilayer periodic structure can be decomposed into stacks of such basic building blocks.

For light collection we want to find out the optimal structure parameters. For each layer, the horizontal thickness is a quarter of the vertical thickness. According to the absorption spectrum of a-Si [13] and the AM $1.5 \mathrm{G}$ solar energy spectrum, the wavelength range of spectrum in our research is from $0.3 \mu \mathrm{m}$ to $0.84 \mu \mathrm{m}$. The simulations 
are done for both polarizations, transverse electric (TE) and transverse magnetic (TM) in the presented results the average values are given.

The complex dielectric constant of metal at optical frequencies is approximated by the Drude model as follows [14]:

$\varepsilon(\omega)=\varepsilon_{0} \varepsilon_{\infty}-\frac{\varepsilon_{0} \omega_{p}^{2}}{\omega^{2}+i \gamma \omega}$

where $\varepsilon_{0}$ is the permittivity of free space, $\varepsilon_{\infty}$ is the dc dielectric constant, $\omega$ is the light frequency, $\omega_{p}$ is the plasma frequency, $\gamma$ is the
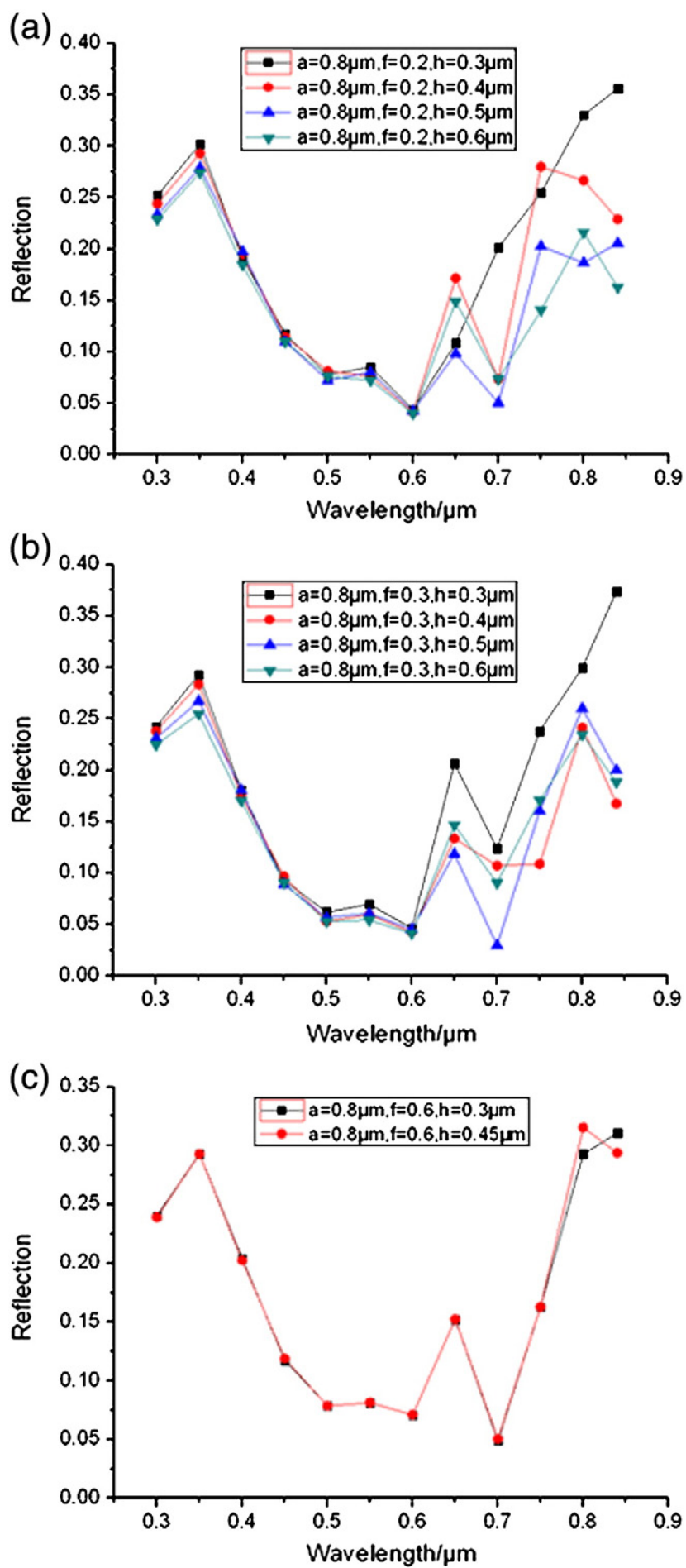

Fig. 6. The reflectivity of the designed TF-Si solar cell as a function of the incident wavelength with the same $a=0.8 \mu \mathrm{m}$, but different $f$ and $h$ for normal incidence. damping frequency. $\omega_{p}=1.3 \times 10^{16} \mathrm{rad} / \mathrm{s}, \quad \gamma=9.6 \times 10^{13} \mathrm{rad} / \mathrm{s}$. For example, at $\lambda=0.8 \mu \mathrm{m}$, the complex dielectric constant of silver is $\varepsilon_{\mathrm{Ag}}=-27.95+1.523 i$, the complex refractive index $n_{\mathrm{Ag}}=0.1439+$ 5.289i. Fig. 2 is the refractive index distribution of the designed TF-Si solar cell at $\lambda=0.8 \mu \mathrm{m}$. We can see that the real part of the complex refractive index of $\mathrm{Ag}$ is 0.1437 , which agrees with our calculated result.

In our simulations, the side wall thickness of each layer is a third of the vertical thickness. The complex refractive index of the materials with different wavelengths is shown in Table 1. $n$ is the refractive index, $\kappa$ is the extinction coefficient. The parameters in Table 1 are obtained from the internet [15]. Both parts of the complex refractive index were considered in simulations for all the layers.

\section{Results and discussion}

Fig. 3 shows the measured reflectivity of a bare silicon wafer $[16,17]$, the simulated reflectivity of a bare Si wafer, and the reflectivity of the flat TF-Si solar cell with different wavelengths for normal incidence. The simulated reflectivity of a bare Si wafer shows excellent agreement with the measured value.

When the incident wavelength is around $0.55 \mu \mathrm{m}$, the reflectivity of the flat TF-Si solar cell is below $10 \%$. But when the incident wavelength is around $0.8 \mu \mathrm{m}$, the reflectivity is higher than $80 \%$. The fluctuation of reflectivity in the wavelength range of $0.6-0.7 \mu \mathrm{m}$ is caused by the interference of light in the multilayer of the TF-Si solar cell.
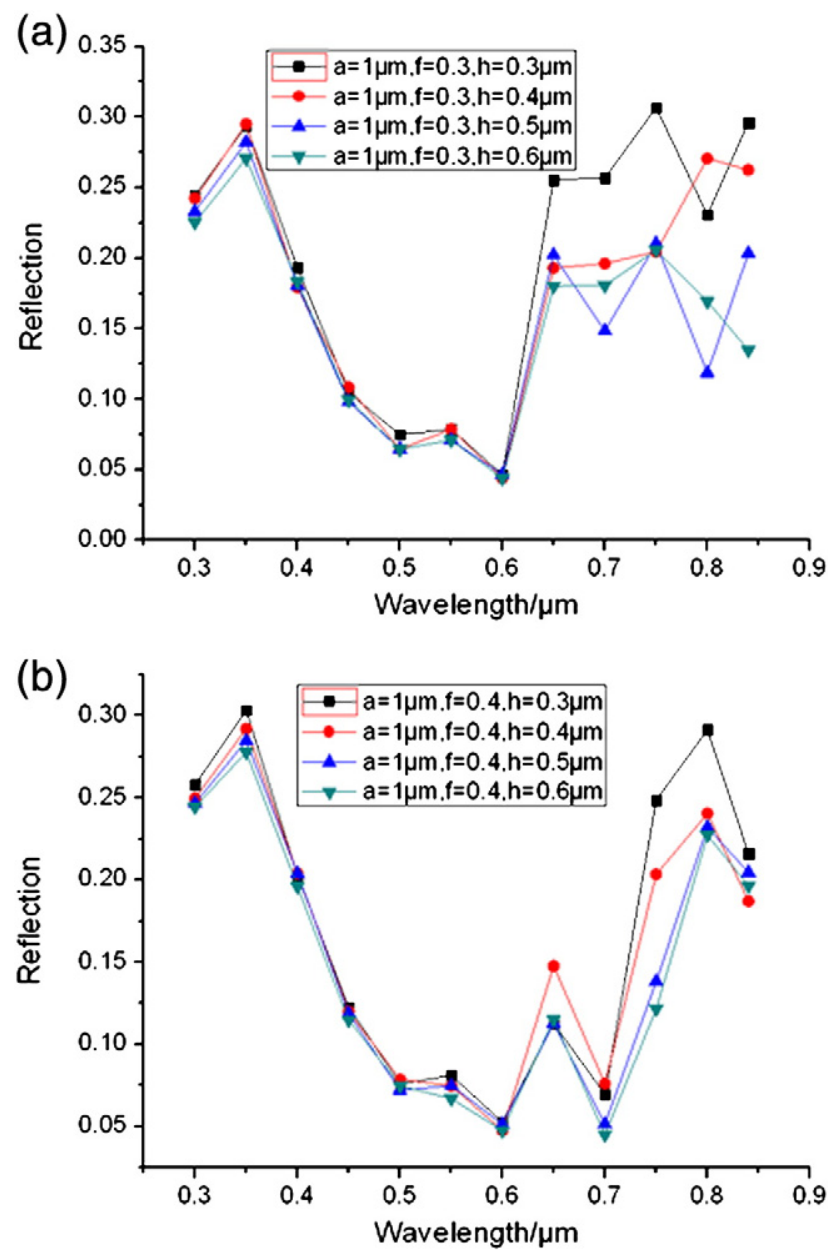

Fig. 7. The reflectivity of the TF-Si solar cell as a function of the incident wavelength with the same $a=1 \mu \mathrm{m}$, but with different $f$ and $h$ for normal incidence. 
Results of simulations of the effects of periodic structures on the net reflection of a-Si solar cells are presented.

When $a=0.5 \mu \mathrm{m}, f=0.1$, the reflectivity of the designed TF-Si solar cell for normal incidence with different $h$ is shown in Fig. 4(a). When $h=0.4 \mu \mathrm{m}$, the reflectivity is the lowest which is from $4 \%$ to $24.4 \%$ in the wavelength range of $0.3-0.65 \mu \mathrm{m}$, reduced by about $28 \%-77 \%$ compared with Fig. 3. In the wavelength range of $0.7-$ $0.84 \mu \mathrm{m}$, the reflectivity with $h=0.4 \mu \mathrm{m}$ is from $1 \%$ to $13.8 \%$.

When $a=0.5 \mu \mathrm{m}, f=0.2$, the reflectivity for normal incidence with different $h$ is shown in Fig. 4(b). When $h=0.4 \mu \mathrm{m}$, the reflectivity is the lowest which is from $3 \%$ to $26 \%$ in the wavelength range of $0.3-0.7 \mu \mathrm{m}$. In the wavelength range of $0.75-0.84 \mu \mathrm{m}$, the reflectivity is from $6 \%$ to $26 \%$ and has no obvious change with $h$. Obviously, when $a=0.5 \mu \mathrm{m}$, we select $f=0.1$ and $h=0.4 \mu \mathrm{m}$.

When $a=0.6 \mu \mathrm{m}, f=0.2$, the reflectivity of the designed TF-Si solar cell for normal incidence with different $h$ is shown in Fig. 5(a). When $h=0.6 \mu \mathrm{m}$, the reflectivity $(1.4 \%-21 \%)$ is almost the lowest in the wavelength range of $0.3-0.84 \mu \mathrm{m}$. In Fig. 5(b), when $a=0.6 \mu \mathrm{m}$, $f=0.3$, the reflectivity has no evident difference with $h$. When $h=0.6 \mu \mathrm{m}$, the reflectivity is almost the lowest, which changes from $3 \%$ to $24.3 \%$ in the wavelength range of $0.3-0.84 \mu \mathrm{m}$. So, when $a=0.6 \mu \mathrm{m}$, we select $f=0.2, h=0.6 \mu \mathrm{m}$.

When $a=0.8 \mu \mathrm{m}, f=0.2$, the reflectivity of the designed TF-Si solar cell for normal incidence with different $h$ is shown in Fig. 6(a). The reflectivity has no obvious change with $h$ in the wavelength range of $0.3-0.6 \mu \mathrm{m}$. When $h=0.5 \mu \mathrm{m}$, the reflectivity is the lowest in the wavelength of $0.6-0.7 \mu \mathrm{m}$ and is from $5 \%$ to $20 \%$ in the wavelength of 0.7-0.84 $\mu \mathrm{m}$. When $a=0.8 \mu \mathrm{m}, f=0.2$, we select $h=0.5 \mu \mathrm{m}$.

When $a=0.8 \mu \mathrm{m}, f=0.3$, the reflectivity of the designed TF-Si solar cell for normal incidence with different $h$ is shown in Fig. 6(b). When $f=0.3$ and $h=0.5 \mu \mathrm{m}$, the reflectivity is $4.5 \%-26.7 \%$ in the wavelength range of $0.3-0.6 \mu \mathrm{m}$, lower than that when $f=0.2$ and $h=0.5 \mu \mathrm{m}$. So when $a=0.8 \mu \mathrm{m}$, we select $f=0.3, h=0.5 \mu \mathrm{m}$.

When $a=0.8 \mu \mathrm{m}, f=0.6$, the reflectivity of the designed TF-Si solar cell for normal incidence with different $h$ is shown in Fig. 6(c). When $a=0.8 \mu \mathrm{m}$ and $f=0.6$, the distance between the adjacent gratings is only $0.1 \mu \mathrm{m}$, which is much smaller than the incident wavelength, just a small amount of light can go through the gap, so the height of the grating has no influence on the reflectivity.

When $a=1 \mu \mathrm{m}$, the reflectivity of the designed TF-Si solar cell for normal incidence with different $f$ and $h$ is shown in Fig. 7. Obviously, when $a=1 \mu \mathrm{m}$, we take $h=0.6 \mu \mathrm{m}$ for both $f=0.3$ and $f=0.4$. When $f=0.4$ and $h=0.6 \mu \mathrm{m}$, the reflectivity is a little higher than that when $f=0.3$ and $h=0.6 \mu \mathrm{m}$ in the wavelength range of $0.3-0.6 \mu \mathrm{m}$, but much lower in the wavelength range of $0.6-0.78 \mu \mathrm{m}$. So when $a=1 \mu \mathrm{m}$, we select $f=0.4, h=0.6 \mu \mathrm{m}$.

Fig. 8 shows the reflectivity of the TF-Si solar cell for different incident angles with the parameters we have selected. Obviously, the reflectivity of the cell with $a=0.5 \mu \mathrm{m}, f=0.1, h=0.4 \mu \mathrm{m}$ is less than $25 \%$ in the whole wavelength range of $0.3-0.84 \mu \mathrm{m}$ within the incident (a)

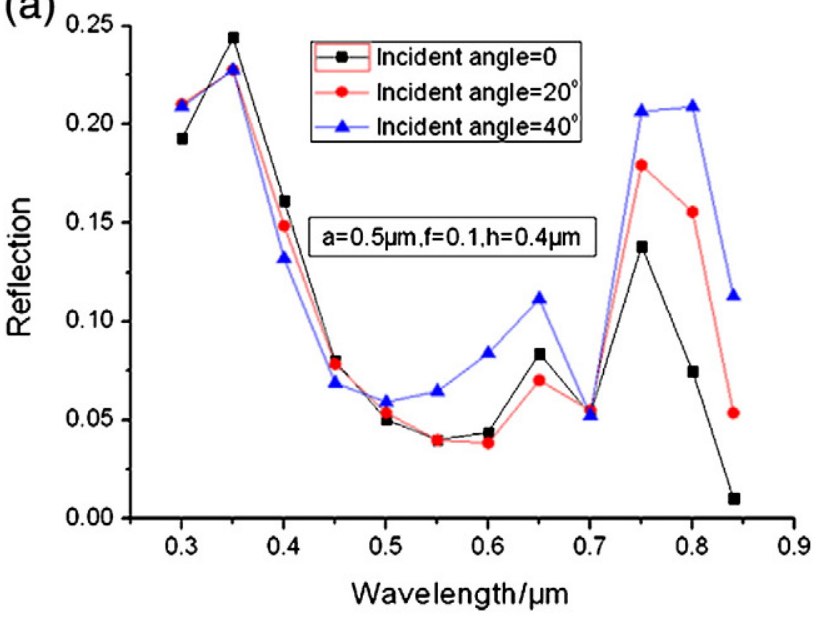

(c) 0

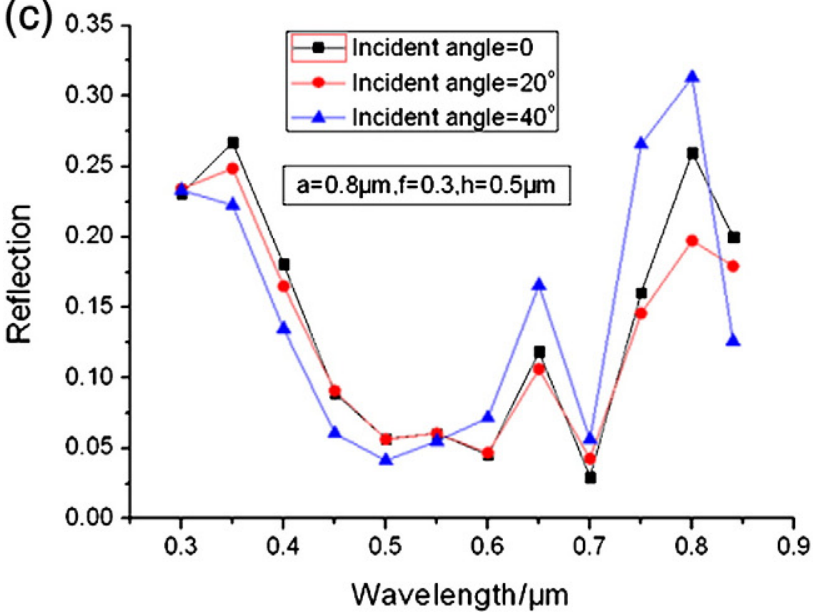

(b)
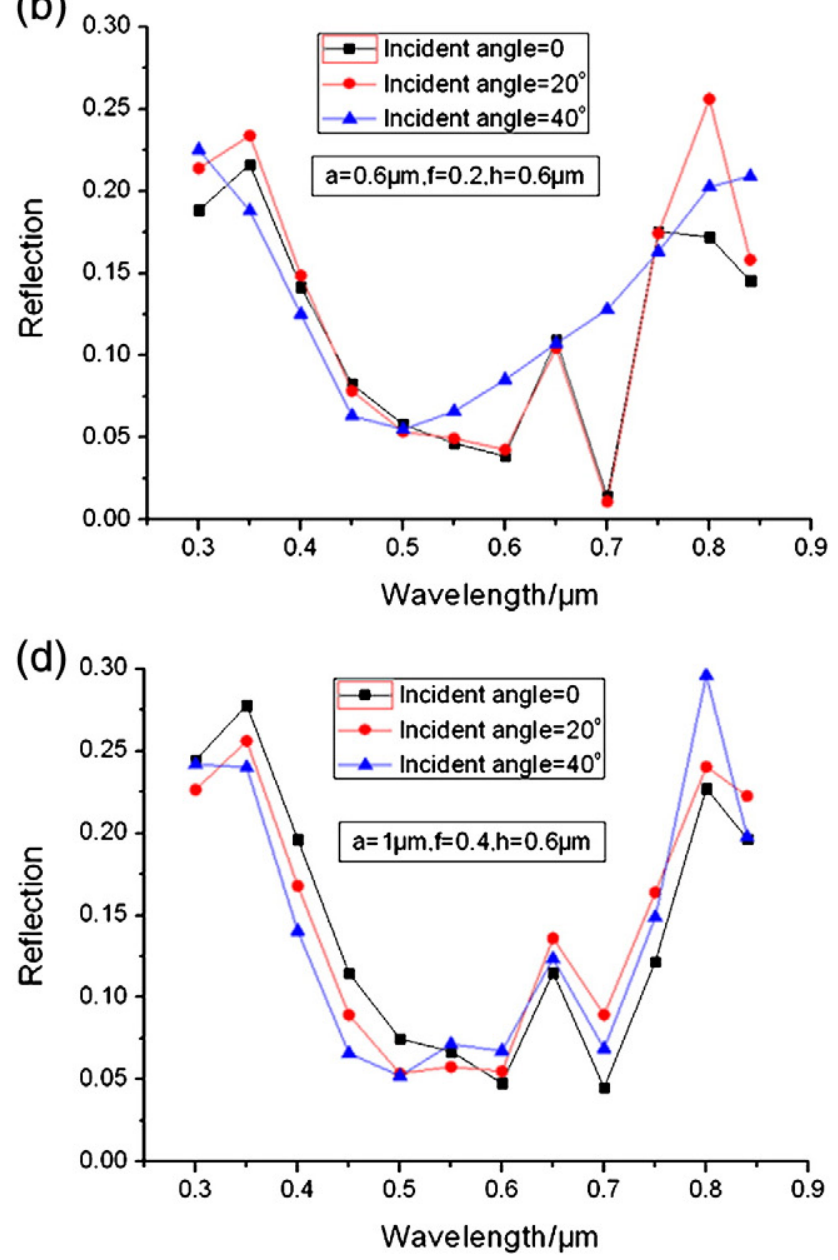

Fig. 8. The reflectivity of the TF-Si solar cell with the structure parameters we have selected for different incident angles. 
(a)

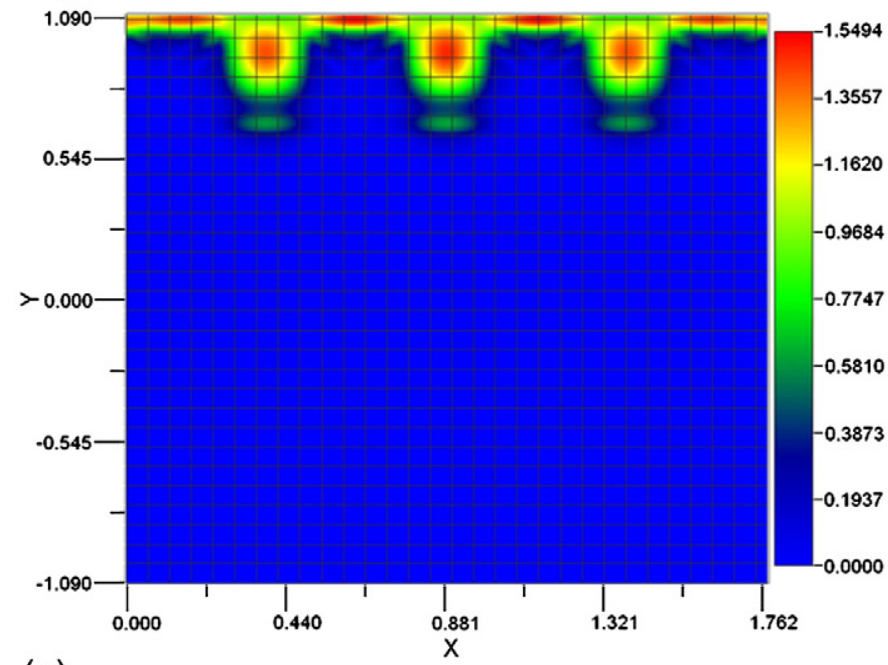

(c)

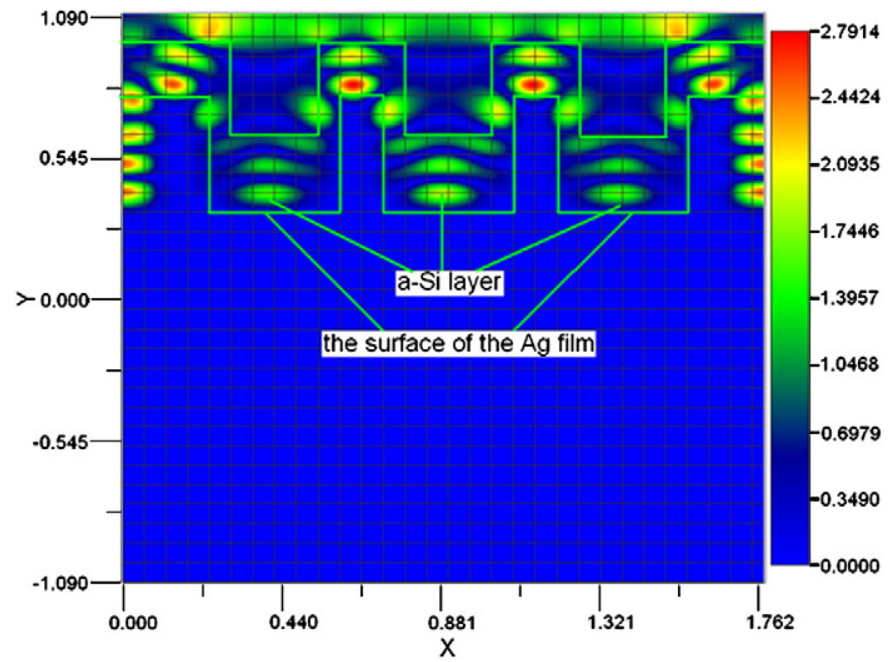

(b)

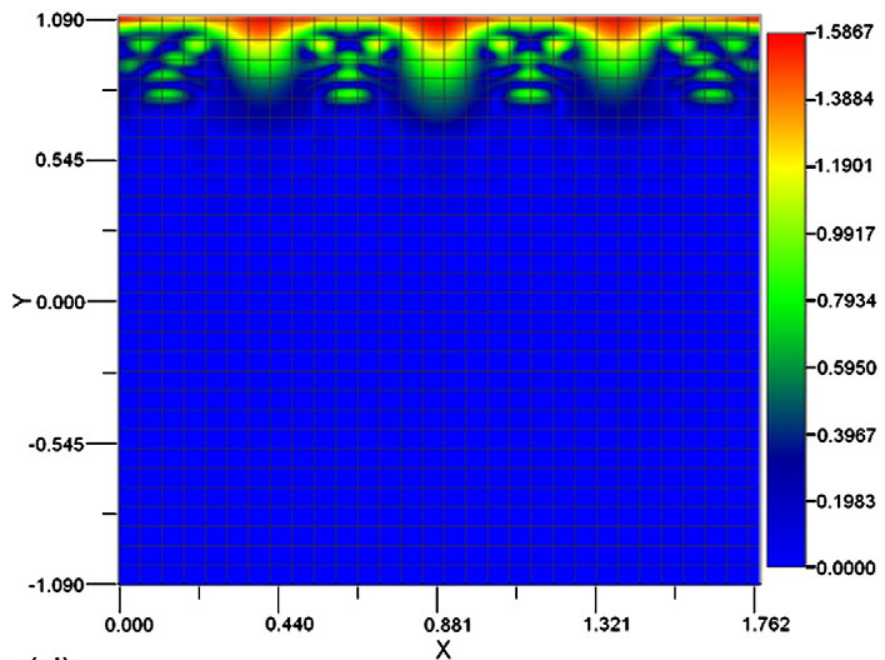

(d)

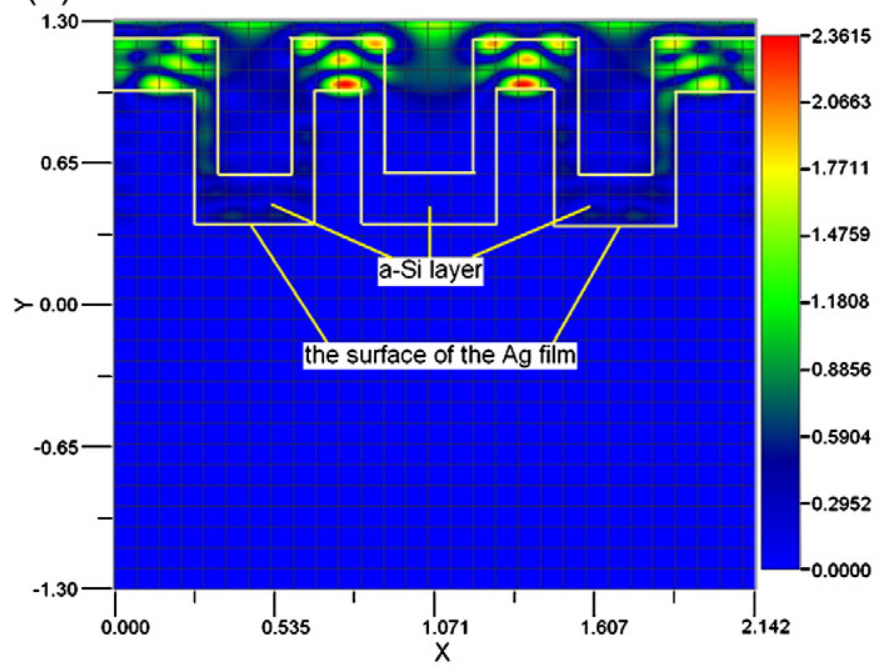

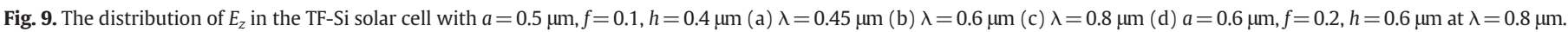

angles of $-40^{\circ}$ to $+40^{\circ}$. Compared with Fig. 3, the reflectivity of the designed cell has been reduced, especially in the wavelength range of $0.6-0.84 \mu \mathrm{m}$. The blue and green light will be absorbed within the first few hundred nanometers of the cell [18,19], so the reflection decrement is about $28-50 \%$. For long wavelengths $(0.6-0.84 \mu \mathrm{m})$, the reflection decrement is about $70 \%-98 \%$. (a)

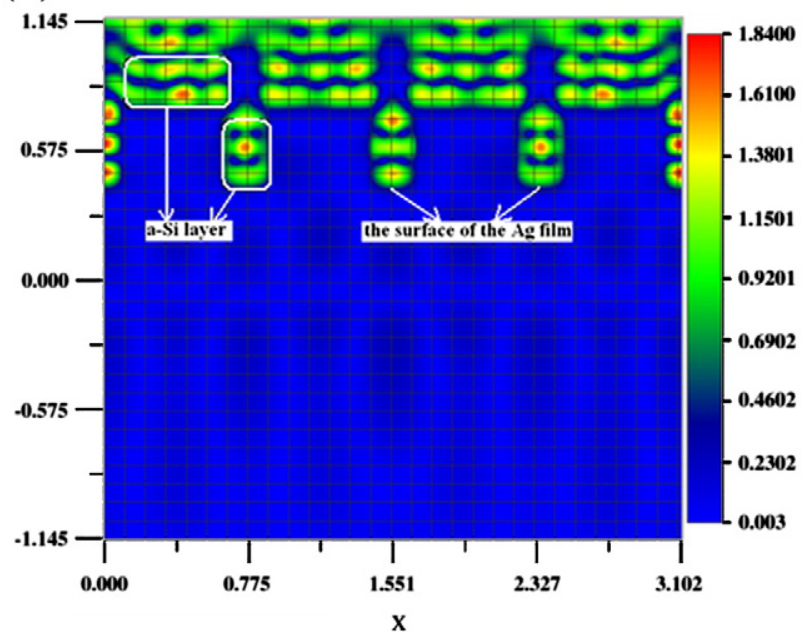

(b)

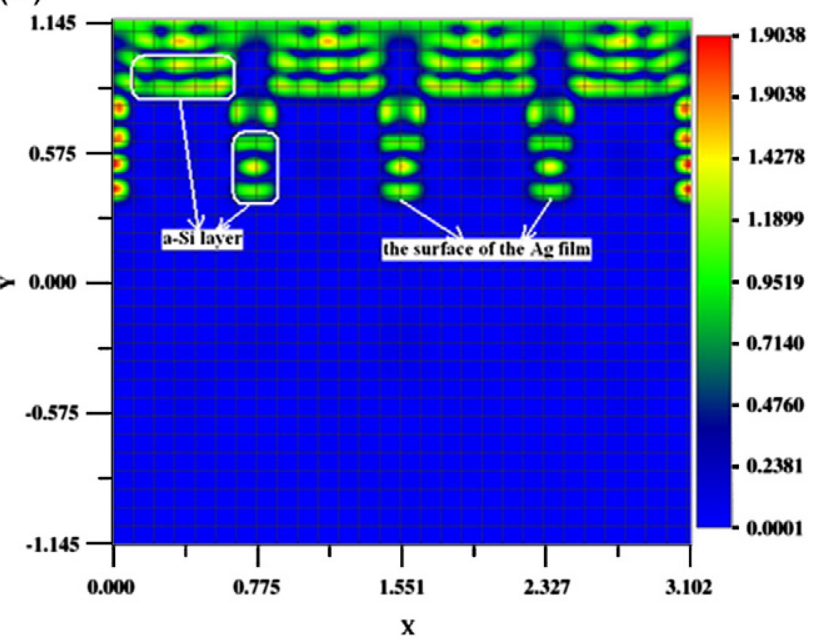

Fig. 10. the distribution of $E_{z}$ in the TF-Si solar cell with $a=0.8 \mu \mathrm{m}, f=0.6, \lambda=0.8 \mu \mathrm{m}$ (a) $h=0.3 \mu \mathrm{m}$ (b) $h=0.45 \mu \mathrm{m}$. 


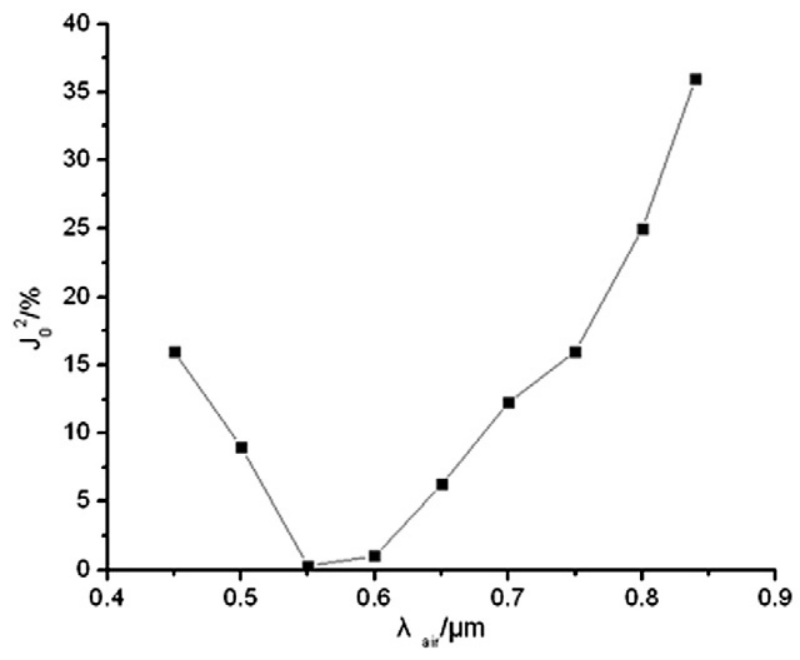

Fig. 11. $J_{0}^{2}$ as a function of $\lambda_{\text {air }}$.

When the period of the grating is comparable to the incident wavelength the diffraction angles are large enough to propagate into their neighboring unit cell. Diffracted waves can thus interface constructively within the thin absorber layer [20]. Based on the above results, we introduce the grating with $a=0.5 \mu \mathrm{m}, f=0.1$, $h=0.4 \mu \mathrm{m}$ on the substrate of the TF-Si solar cell.

Fig. 9 shows the electric field $E_{z}$ distribution inside the TF-Si solar cell with $a=0.5 \mu \mathrm{m}, f=0.1, h=0.4 \mu \mathrm{m}$ for normal incidence. In the case of short wavelength illumination $(\lambda=0.45$ and $0.6 \mu \mathrm{m})$ photons get absorbed within the first few hundred nanometers of the cell. The high absorption coefficient of silicon for wavelengths 0.45 and $0.6 \mu \mathrm{m}$ inhibits the propagating wave to hit the back reflector. In the case of long wavelength illumination $(\lambda=0.8 \mu \mathrm{m})$, diffraction from the front makes more light into the a-Si absorber layer, back metallic grating reflects the incoming light at an oblique angle and bounds the light in the absorber layer toward higher absorption, as shown in Fig. 9(c). Fig. 9 (d) is the distribution of $E_{z}$ in the cell with $a=0.6 \mu \mathrm{m}, f=0.2$, $h=0.6 \mu \mathrm{m}$. Obviously, when $a=0.5 \mu \mathrm{m}, f=0.1, h=0.4 \mu \mathrm{m}$, the electric field intensity in a-Si layer is stronger than that when $a=0.6 \mu \mathrm{m}$, $f=0.2, h=0.6 \mu \mathrm{m}$. When the designed TF-Si solar cell has the lowest reflection, the electric field strength in the a-Si layer is the strongest.

In Fig. 6 (c) when $a=0.8 \mu \mathrm{m}$ and $f=0.6$, the height of the grating has no obvious influence on the reflectivity. In Fig. 10, when $h=0.3 \mu \mathrm{m}$ and $0.45 \mu \mathrm{m}$, the light quantity and light intensity in the a-Si layer is almost the same. Fig. 10 shows good agreement with Fig. 6(c).

Effect other than reflection on the periodically modulated back reflector must play an important role. Now, the effect of the grating at the air/ITO interface cannot been neglected. This interface will act as a phase grating. It is known that phase gratings can be very effective in the suppression of the 0 th diffraction order [21]. The surface reflectivity could be roughly calculated according to the zeroth order diffraction efficiency, which can be estimated by the square of the Bessel function $J_{0}(\pi \Delta \phi)[20] . \Delta \phi$ is the peak to peak phase shift. When $a=0.5 \mu \mathrm{m}$, $f=0.1$, and $h=0.4 \mu \mathrm{m}, \Delta \phi$ for a wavelength can be calculated according to

$\Delta \phi=\frac{h}{\lambda_{\text {air }}}\left(n_{\text {ITO }}-n_{\text {air }}\right)$

$J_{0}^{2}$ with different wavelengths is shown in Fig. 11 . The change regularity of Fig. 11 is similar with that of Fig. 4(a).

Fig. 12 shows the absorption of the a-Si solar cell. With the periodic grating ( $a=0.5 \mu \mathrm{m}, f=0.1, h=0.4 \mu \mathrm{m}$ ) on the substrate, the absorption of the a-Si solar cell has been enhanced, especially in the wavelength range of $0.6-0.84 \mu \mathrm{m}$. The blue and green light will be absorbed within

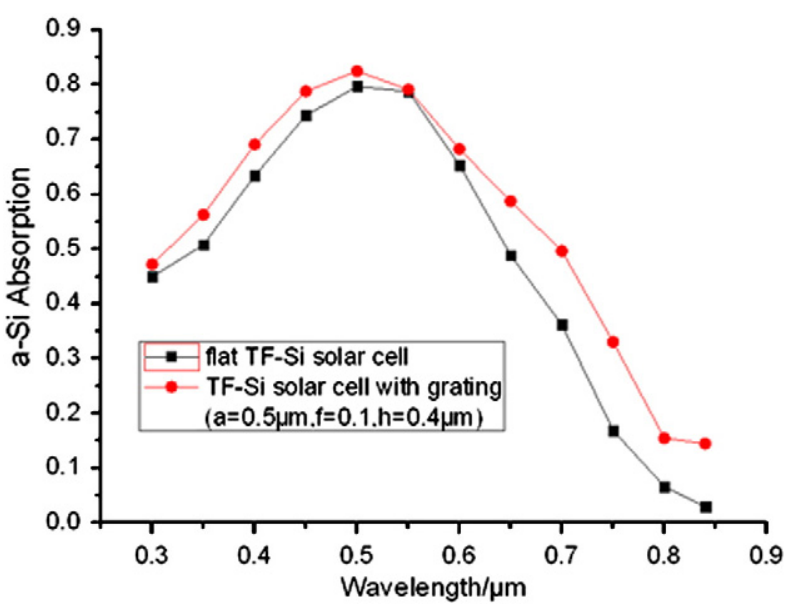

Fig. 12. The absorption of the a-Si solar cell.

the first few hundred nanometers of the cell [17], so in the wavelength range of $0.3-0.6 \mu \mathrm{m}$, the absorption enhancement is only about $0.4 \%-10.8 \%$. For long wavelengths $(0.6-0.84 \mu \mathrm{m})$, the absorption enhancement is about $20 \%-385 \%$.

\section{Conclusion}

We have presented our design of submicron surface texture in amorphous TF-Si solar cells on plastic foil. We identified the periodic structures on plastic substrate for both top anti-reflection and back reflector. The reflectance of a-Si:H solar cells with periodic gratings was investigated by means of optical simulations. The reflectivity of the TF-Si solar cell with a period of $0.5 \mu \mathrm{m}$, a filling factor of 0.1 and a groove depth of $0.4 \mu \mathrm{m}$ is reduced by $28 \%-98 \%$ in the wavelength range of $0.3-0.84 \mu \mathrm{m}$ and within the incident angles of $-40^{\circ}$ to $+40^{\circ}$. The absorption of the a-Si solar cell with the gratings is enhanced by $0.4 \%-10.8 \%$ in the wavelength range of $0.3-0.6 \mu \mathrm{m}$ and $20 \%-385 \%$ in the wavelength range of $0.6-0.84 \mu \mathrm{m}$.

\section{Acknowledgements}

This research was supported by the projects of The Seventh Framework Programme Seventh Framework Programme (FP7) of European Commission (ENERGY FP7-ENERGY-2009-1: Silicon-Light, Grant no. 241277-2) and National High-Tech Research and Development Program of China (Grant No. 2011AA050518).

\section{References}

[1] B. Rech, H. Wagner, Applied Physics A Materials Science Process 69 (2) (1999) 155.

[2] J. Meier, J. Spitznagel, U. Kroll, C. Bucher, S. Faÿ, T. Moriarty, A. Shah, Thin Solid Films 451-452 (2004) 518.

[3] P. Pernet, R. Felder, M. Goetz, H. Keppner, D. Fischer, A. Shah, 14th EC Photovoltaic Solar Energy Conference, 1997, p. 2339.

[4] F.J. Haug, T. Söderström, M. Python, V. Terrazzoni-Daudrix, X. Niquille, C. Ballif, Solar Energy Materials and Solar Cells 90 (6-7) (2009) 884.

[5] J. Löffler, L.A. Wipliez, M.A. Keijzer, J. Bosman, W.J. Soppe, Presented at The International Congress on Applications of Lasers \& Electro-Optics (ICALEO), Orlando, USA, 2-5 November, 2009, 2009.

[6] M. Heijna, J. Löffler, B.B. Aken, W.J. Soppe, Presented at SPIE Optics \& Photonics Conference, San Diego, USA, 12-14 August, 2008, 2008.

[7] H. Gleskova, I.C. Cheng, S. Wagner, J.C. Sturm, Z.G. Suo, Solar Energy 80 (6) (2006) 687.

[8] W.J. Soppe, B.B. Van Aken, M. Dörenkämper, C. Devilee, M.C.R. Heijna, J. Löffler, 24th European Photovoltaic Solar Energy Conference and Exhibition, 21-25 September 2009, Hamburg, Germany, 2009.

[9] B. Sopori, J. Madjdpour, Y. Zhang, W. Chen, S. Guha, J. Yang, A. Banerjee, S. Hegedus, Materials Research Society Symposium Proceedings 557 (1999) 755.

[10] J. Müller, B. Rech, J. Springer, M. Vanecek, Solar Energy 77 (6) (2004) 917.

[11] U. Manna, J. Yoo, S.K. Dhungel, M. Gowtham, U. Gangopadhyay, K. Kim, J. Yi, Journal of Korean Physics Society 46 (6) (2005) 1378. 
[12] H.J. Zhao, Y.J. Peng, J. Tan, C.R. Liao, P. Li, X.X. Ren, Chinese Physics B 18 (2009) 5326.

[13] R. Santbergen, J.M. Goud, M. Zeman, J.A.M. Roosmalen, R.J.C. Zolingen, Solar Energy Materials and Solar Cells 94 (5) (2010) 715.

[14] M.C. Quong, A.Y. Elezzabi, Optics Express 15 (16) (2007) 10163.

[15] http://refractiveindex.info/?gclid=CIX-9te-v6ICFQ0YewodAx9h4w.

[16] Z.N. Yu, H. Gao, W. Wu, H.X. Ge, S.Y. Chou, Journal of Vacuum Science and Technology B 21 (6) (2003) 2874.

[17] W.D. Zhou, M. Tao, L. Chen, H.J. Yang, Journal of Applied Physics 102 (10) (2007) 103.
[18] R. Dewan, M. Marinkovic, R. Noriega, S. Phadke, A. Salleo, D. Knipp, Optics Express 17 (25) (2009) 23058

[19] L. Zhao, Y.H. Zuo, C.L. Zhou, H.L. Li, H.W. Diao, W.J. Wang, Solar Energy 84 (1) (2010) 110

[20] M.T. Gale, Optics Communications 18 (3) (1976) 292.

[21] C. Eisele, C.E. Nebel, M. Stutzmann, Journal of Applied Physics 89 (12) (2001) 7722. 